Supplementary videos

Video 1 caption: Video capsule endoscopy staging Peutz-Jeghers disease.

Pedunculated polyp in the stomach.

Video 2 caption: Video capsule endoscopy staging Peutz-Jeghers disease.

Pedunculated polyp in the proximal jejunum

\title{
Peutz-Jeghers syndrome: capsule endoscopy to stage disease
}

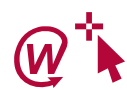

Henedina Antunes, João Nascimento, Paula Peixoto

A 13-year-old girl with recurrent abdominal pain and rectal bleeding was referred to our department. Her 36-year-old father had been diagnosed with Peutz-Jeghers syndrome (PJS) after previous surgical interventions for intestinal occlusion. On physical examination, she had hyperpigmented macules on her lips and buccal mucosa. Genetic testing showed a mutation in exon 6 of the gene STK11. A video capsule endoscopy was done to stage the disease. A $7 \mathrm{~mm}$ polyp in the stomach (figure A; video 1), and a $12 \mathrm{~mm}$ pedunculated polyp in the proximal jejunum, were seen (figure B; video 2). Smaller polyps were found sparsely in the jejunum and proximal ileum. Screening and surveillance is crucial in Peutz-Jeghers syndrome because of the risk of complications related to polyps, and the association with cancers. Our patient's case shows the feasibility, security, and sensitivity of use of capsule endoscopy to stage disease.


Figure: Peutz-Jeghers syndrome

Video capsule endoscopy showing (A) pedunculated polyp in the stomach; and (B) pedunculated polyp in the proximal jejunum.
Published Online

October 5, 2012

http://dx.doi.org/10.1016/

S0140-6736(12)60830-7

Gastroenterology, Hepatology and Nutrition Unit, Department of Pediatrics

(Prof H Antunes MD, J Nascimento MD) and Department of Gastroenterology (P Peixoto MD), Braga Hospital, Braga, Portugal; Life and Health Sciences Research Institute (ICVS), School of Health Sciences, University of Minho, Braga, Portugal (Prof H Antunes); and ICVS/3B's_-PT Government Associate Laboratory, Braga/Guimarães, Portugal (Prof H Antunes)

Correspondence to: Prof Henedina Antunes,

Gastroenterology, Hepatology and Nutrition Unit, Departmen of Pediatrics, Hospital de Braga Apartado 2056, 4701-901 Braga, Portugal

henedinaantunes@gmail.com 